\title{
STRUCTURE OF OPTIMAL TRAJECTORIES IN A NONLINEAR DYNAMIC MODEL WITH ENDOGENOUS DELAY
}

\author{
NATALI HRITONENKO AND YURI YATSENKO
}

Received 12 November 2003 and in revised form 16 February 2004

An exact solution is constructed to a nonlinear optimization problem in an integral dynamic model with delay. The problem involves the unknown duration of the delay and has important applications to the optimal replacement of capital equipment under technological change.

\section{Introduction}

One of the modern applications of integral equations is the replacement of capital equipment under technological change. Corresponding models are known as vintage capital models (VCMs), see $[1,2,3,4,5,13,14,17]$. They focus on optimization of the equipment lifetime and can be expressed via special Volterra integral equations with delay (e.g., Corduneanu [5]). Existing results about endogenous equipment lifetime in VCMs include mainly the case of constant lifetime.

This paper is devoted to the construction of exact solutions to an optimization problem (OP) with endogenous equipment lifetime in the well-known Solow VCM [16]. The authors investigated the integral models with endogenous delay for various applied problems of economics, ecology, and engineering (see $[6,7,8,9,10,12,19,20]$ and the references therein). They provided an asymptotic analysis of the OP under study and discovered turnpike properties of the optimal equipment lifetime in [21] (see also [7, 8]). More complicated models with many inputs and outputs were investigated in $[7,10,19]$.

The paper is organized as follows. The OP for the Solow model with endogenous capital lifetime is formulated in Section 2. Section 3 exposes preliminary results such as the condition for an extremum, gradient of the OP, and arising auxiliary nonlinear integral equation. In Section 4, the exact structure of optimal trajectories is established.

\section{Statement of optimization problem}

The OP under study consists of finding the functions $m(t)$ and $a(t), t \in\left[t_{0}, T\right], T<\infty$, which maximize the objective functional 


$$
I=\int_{t_{0}}^{T} \rho(t)\left[\int_{a(t)}^{t} \beta(\tau, t) m(\tau) d \tau-\lambda(t) m(t)\right] d t \longrightarrow \max _{a, m}
$$

under the constraint equality

$$
P(t)=\int_{a(t)}^{t} m(\tau) d \tau
$$

the constraint inequality

$$
m_{\min }(t) \leq m(t) \leq M(t), \quad \text { where } m_{\min }(t)=\max \left\{0, P^{\prime}(t)\right\}
$$

and the initial conditions

$$
a\left(t_{0}\right)=a_{0}<t_{0}, \quad m(\tau)=m_{0}(\tau), \quad \tau \in\left[a_{0}, t_{0}\right]
$$

In mathematical economics, OP (2.1)-(2.4) describes the maximization of the net revenue (output minus investments) of an economic system in the Solow VCM [16]. The unknown variables are the investment $m(t)$ and the scrapping time $a(t)$ of obsolete capital, $t \in\left[t_{0}, T\right]$. Then $t-a(t)$ is the endogenous lifetime of the capital (the age of the oldest equipment still in use). The given characteristics are the specific productivity $\beta(\tau, t)$ (output per one worker on the equipment introduced at time $\tau$ ), the specific cost $\lambda(t)$ of new equipment (per one worker), the total labour $P(t)$, the discounting factor $\rho(t)$, $0<\rho(t) \leq 1, \rho^{\prime}(t) \leq 0, t \in\left[t_{0}, T\right]$, and the investments $m_{0}(\tau)$ made on the prehistory interval $\left[a\left(t_{0}\right), t_{0}\right]$. The productivity $\beta(\tau, t)$ represents the technological change embodied in the new equipment vintages and strictly increases in $\tau$ (new machines are more efficient than the older ones).

We assume that the given functions $\beta, \lambda, P, \rho$, and $M$ are Lipschitz continuous, $m_{0}$ is piecewise continuous, all these functions are positive and satisfy (2.2)-(2.4) at $t=t_{0}$.

\section{Preliminary results}

Presence of the unknown function $a$ in integration limits determines the novelty of the OP. The investigation methods for such OPs were developed by Hritonenko and Yatsenko [7] and are based on common variation (perturbation) techniques of optimization theory (see, e.g., $[11,15,18]$ ). We introduce the gradient of functional (2.1) and express the extremum conditions in terms of the gradient.

3.1. The OP gradient. Let $m(t), t \in\left[t_{0}, T\right]$, be the independent control variable of $\mathrm{OP}$ (2.1)-(2.4). Then the function $a(t), t \in\left[t_{0}, T\right]$, is a dependent (phase) variable. As shown in Yatsenko [19], for any measurable control $m$ that satisfies (2.3) almost everywhere (a.e.) on $\left[t_{0}, T\right]$, a unique a.e. continuous function $a(t)<t, t \in\left[t_{0}, T\right]$, exists which satisfies (2.2), (2.4) and a.e. has $a^{\prime}(t) \geq 0$. In other words, the scrapping time $a(t)$ is monotonic and the scrapped equipment cannot be used again. The set of the measurable variables $m$ that satisfy condition (2.3) is denoted by $\mathbf{U}$. 
As shown in Hritonenko and Yatsenko [7], the increment $\delta I$ of functional (2.1) in OP (2.1)-(2.4) is of the form

$$
\delta I=I(m+\delta m)-I(m)=\int_{t_{0}}^{T} I^{\prime}(t) \delta m(t) d t+\delta^{2} I,
$$

where the gradient of functional I is

$$
\begin{aligned}
I^{\prime}(t) & =\int_{t}^{\bar{a}^{-1}(t)} \rho(\tau)[\beta(t, \tau)-\beta(a(\tau), \tau)] d \tau-\lambda(t) \rho(t), \quad t \in\left[t_{0}, T\right), \\
\bar{a}^{-1}(t) & = \begin{cases}a^{-1}(t), & t \in\left[t_{0}, a(T)\right], \\
T, & t \in[a(T), T),\end{cases}
\end{aligned}
$$

$a^{-1}(t)$ is the inverse function of $a(t)$, and the higher-order variation residual is

$$
\delta^{2} I=\int_{t_{0}}^{T} \rho(t) \int_{a(t)}^{a(t)+\delta a(t)}[\beta(a(t), t)-\beta(\tau, t)][m(\tau)+\delta m(\tau)] d \tau d t=O\left(|\delta m|^{2}\right)
$$

According to (2.2), the admissible variations $\delta m(t), \delta a(t), t \in\left[t_{0}, T\right]$, of the functions $m(t), a(t), t \in\left[t_{0}, T\right]$, in formulas (3.1)-(3.3) satisfy the equality

$$
\begin{aligned}
\int_{\max ^{\left\{a(t), t_{0}\right\}}}^{t} \delta m(\tau) d \tau & =\int_{a(t)}^{a(t)+\delta a(t)}\left[m(\tau)+\delta m_{\mathrm{int}}(\tau)\right] d \tau, \\
\delta m_{\mathrm{int}}(\tau) & = \begin{cases}\delta m(\tau), & t \in\left(t_{0}, T\right), \\
0, & t \in\left[a\left(t_{0}\right), t_{0}\right] .\end{cases}
\end{aligned}
$$

3.2. The necessary and sufficient condition for an extremum. In order for a function $m^{*}(t), t \in\left[t_{0}, T\right]$, to be a solution of OP (2.1)-(2.4), it is necessary and sufficient that

$$
\begin{gathered}
I^{\prime}\left(a^{*} ; t\right)<0 \quad \text { at } m^{*}(t)=m_{\min }(t), \\
I^{\prime}\left(a^{*} ; t\right)>0 \quad \text { at } m^{*}(t)=M(t), \\
I^{\prime}\left(a^{*} ; t\right) \equiv 0 \quad \text { at } m_{\min }(t) \leq m^{*}(t) \leq M(t), t \in\left[t_{0}, T\right] .
\end{gathered}
$$

The proof is given by Hritonenko and Yatsenko [7, 8]. The proof of the necessary condition is standard for such OPs. The sufficiency follows from the convexity of the functional $I(m)$ that holds because $\beta(\tau, t)$ is monotonic in $\tau$. We will now illustrate it. Using the mean value theorem, (3.3) can be rewritten as

$$
\delta^{2} I=\int_{t_{0}}^{T} \rho(t)[\beta(a(t), t)-\beta(a(t)+\chi(t), t)] \int_{a(t)}^{a(t)+\delta a(t)}[m(\tau)+\delta m(\tau)] d \tau d t
$$


where $0<\chi(t)<\delta a(t)$. Let $\delta m(\tau)=m_{1}(\tau)-m_{2}(\tau) \geq 0, \tau \in\left[t_{0}, T\right)$, and $\delta m(\tau)>0, \tau \in$ $\Delta_{m} \subset\left[t_{0}, T\right)$. Then, in view of (3.4), the corresponding variation $\delta a(t) \geq 0$ at $t \in\left[t_{0}, T\right)$ and $\delta a(t)>0$, at least, for $t \in \Delta=\left\{t: \Delta_{m} \cap[a(t), t] \neq \varnothing\right\}$, and $\int_{a(t)}^{a(t)+\delta a(t)}[m(\tau)+$ $\delta m(\tau)] d \tau \geq 0$ at $t \in\left[t_{0}, T\right)$ and is positive on $\Delta$. Also, $\beta(a(t), t)-\beta(a(t)+\chi(t), t)<0$. Hence, the integrand in the last formula for $\delta^{2} I$ is nonpositive on $\left[t_{0}, T\right)$ and is negative on some subset $\Delta$ of $\left[t_{0}, T\right)$, that is, $\delta^{2} I<0$. The case $\delta m(\tau)<0$ leads to the same result. Therefore, the functional $I(m)$ is strictly convex.

If $m(t)=0$ at some points $t \in\left[t_{0}, T\right)$, then in view of (2.2) the variation $\delta a(t)$ can be finite for an infinitesimal $\delta m(\tau), \tau<t$. In this case, the functional $I(m)$ is not differentiable, and expression (3.2) does not represent the gradient of functional (2.1). However, conditions (3.5) are still valid in this case because of the convexity of the functional $I(m)$ $[14,15]$. The case $m=0$ is natural in economics and is also presented below.

3.3. Dual integral-functional equation. As follows from (3.5), the integral-functional equation $I^{\prime}(a ; t)=0, t \in\left[t_{0}, T\right), T \leq \infty$, or

$$
\int_{t}^{a^{-1}(t)} \rho(\tau)[\beta(t, \tau)-\beta(a(\tau), \tau)] d \tau=\lambda(t) \rho(t), \quad t \in\left[t_{0}, T\right)
$$

with respect to the unknown function $a$ plays an important role in a qualitative analysis of the OP solutions. In accordance with the economic content, we consider only its monotonic solutions $a(t)<t$.

Equation (3.7) generates a set of solutions $a_{T}(t)$ for a finite interval $\left[t_{0}, T\right]$. The given functions $\beta, \lambda, \rho$ need to satisfy some strict conditions for the existence of the solutions $a_{T}$ on large intervals $\left[t_{0}, T\right], T \gg t_{0}$. The existence and uniqueness of the infinite solution $\tilde{a}(t), t \in\left[t_{0}, \infty\right)$, has been investigated in $[6,7]$ for various combinations of exponential, power, and logarithmic functions $\beta$ and $\lambda$.

Here and thereafter we assume that

$$
\begin{gathered}
\beta(\tau, t)=\beta_{0} \exp \left(c_{1} \tau\right), \quad \lambda(t)=\lambda_{0} \exp \left(c_{2} t\right), \quad \rho(t)=\exp \left(-c_{3} t\right), \\
c_{1}, \beta_{0}, \lambda_{0}>0, \quad c_{2} \leq c_{1}<c_{3}, \quad \beta_{0}\left(c_{3}-c_{1}\right)>\lambda_{0} c_{3}\left(c_{3}-c_{2}\right) \exp \left[\left(c_{2}-c_{1}\right) t_{0}\right] .
\end{gathered}
$$

According to $[6,7],(3.7)$ has a unique solution $\tilde{a}(t), d \tilde{a} / d t>0, t \in\left[t_{0}, \infty\right)$, such that

(1) if $c_{1}>c_{2}$, then $t-\tilde{a}(t) \rightarrow 0$ at $t \rightarrow \infty$;

(2) if $c_{1}=c_{2}$, then $\tilde{a}(t) \equiv t-L, t \in\left[t_{0}, \infty\right)$, where the constant $L$ is determined from the following nonlinear equation

$$
c_{3} \exp \left(-c_{1} L\right)-c_{1} \exp \left(-c_{3} L\right)=\left(c_{3}-c_{1}\right)\left(1-\frac{c_{3} \lambda_{0}}{\beta_{0}}\right) .
$$

In particular, $L \approx\left[2 \lambda_{0} / \beta_{0} / c_{1}\right]^{1 / 2}$ for $0 \leq c_{1}<c_{3} \ll 1$. Equation (3.7) has also a set of solutions $a_{T}(t), d a_{T} / d t>0$, for any interval $t \in\left[t_{0}, T\right]$ such that all the solutions $a_{T}(\cdot)$ approach the unique solution $\tilde{a}(\cdot)$ at $T-t_{0} \rightarrow \infty$. 
3.4. Asymptotics of OP solutions. The study of the asymptotic behavior of OP solutions at large $T-t_{0} \gg 1$ was provided by Yatsenko [19], Hritonenko and Yatsenko [7, 8, 10] where a convergence of the optimal trajectories $a^{*}$ to (3.7) solution $\tilde{a}$ on the infinite interval $\left[t_{0}, \infty\right)$ was established. Namely, under some assumptions $[7,8]$, the solution $a^{*}$ to OP (2.1)-(2.4) strives to $\tilde{a}$ at $T \rightarrow \infty$ on an asymptotically largest part of the interval $\left[t_{0}, T\right]$, that is, for any $\varepsilon>0$, the time $T_{0}$ exists such that for any $T \geq T_{0}$ the condition $\left|a^{*}(t)-\tilde{a}(t)\right|<\varepsilon$ is true on some subset $\Delta \subset\left[t_{0}, T\right]$ such that mes $(\Delta) /\left(T-t_{0}\right) \rightarrow 1$ for $T \rightarrow \infty$. In economics, such phenomena are known as turnpike properties.

Next, the exact structure of the solution $\left(m^{*}, a^{*}\right)$ of OP (2.1)-(2.4), (3.8) is studied.

\section{Structure of OP solutions}

Lemma 4.1. There is an instant $\Theta, t_{0} \leq \Theta<T$, such that $I^{\prime}\left(a^{*} ; t\right)<0$ and the OP solution is the minimum possible from $\Theta$ onward: $m^{*}(t) \equiv m_{\min }(t), a^{*}(t) \equiv a_{\min }(t)$ for $t \in(\Theta, T]$, where $m_{\min }$ is determined by (2.3).

The proof follows directly from the analysis of expression (3.2) at $t$ close to $T$. Lemma 4.1 shows that the OP (2.1)-(2.4), (3.8) possesses a "zero-investment period" at the end of the planning horizon, which is a common effect in various finite-horizon OPs of mathematical economics.

We construct an exact analytic solution to the nonlinear OP (2.1)-(2.4), (3.8). The technique is essentially based on Lemma 4.1 and the special structure of the expression (3.2) for the gradient $I^{\prime}(a ; t), t \in[t, T]$. Namely, $I^{\prime}(a ; t)$ does not depend explicitly on the independent unknown control $m$. It allows us to use the following approach to construct the OP solution. We start the construction of the solution $a^{*}$ from the right end of the horizon $\left[t_{0}, T\right]$ because Lemma 4.1 gives the clear clue about its behavior: $a^{*}(t)=a_{\min }(t)$ on some interval $(\Theta, T], \Theta \geq t_{0}$. Then, if $\Theta>t_{0}$, we try to build the solution $a^{*}$ recurrently from the right to the left, adjusting $I^{\prime}\left(a^{*} ; t\right)$ to zero and keeping its value zero where it is possible. The corresponding solution $m^{*}(t), t \in\left[t_{0}, T\right]$, is determined from (2.2). Finally, we will verify that the constructed solution satisfies the extremum conditions (3.5).

4.1. Analysis of dual equation. In order to implement the above-described structure of the OP solution, we need to solve equation $I^{\prime}(a ; t) \equiv 0$ on the interval $\left[t_{0}, T\right]$ with the initial condition at the right end:

$$
a(t) \equiv a(\Theta)=\text { const }, \quad t \in(\Theta, T] .
$$

In case (3.8), the OP gradient (3.2) is determined as

$$
I^{\prime}(a ; t)=\beta_{0} \int_{t}^{\bar{a}^{-1}(t)} e^{-c_{3} \tau}\left[e^{c_{1} t}-e^{c_{1} a(\tau)}\right] d \tau-\lambda_{0} e^{\left(-c_{3}+c_{2}\right) t}, \quad t \in\left[t_{0}, T\right] .
$$

The differentiation of equation $I^{\prime}(a ; t) \equiv 0$ leads to the following expression:

$$
t-a(t)=-\frac{1}{c_{1}} \ln \left\{\frac{1-\lambda_{0}}{\beta_{0}\left(c_{3}-c_{2}\right) e^{\left(c_{2}-c_{1}\right) t}+\left(c_{1} / c_{3}\right)\left[e^{-c_{3}\left(\bar{a}^{-1}(t)-t\right)}-1\right]}\right\}, \quad t \in \Delta .
$$




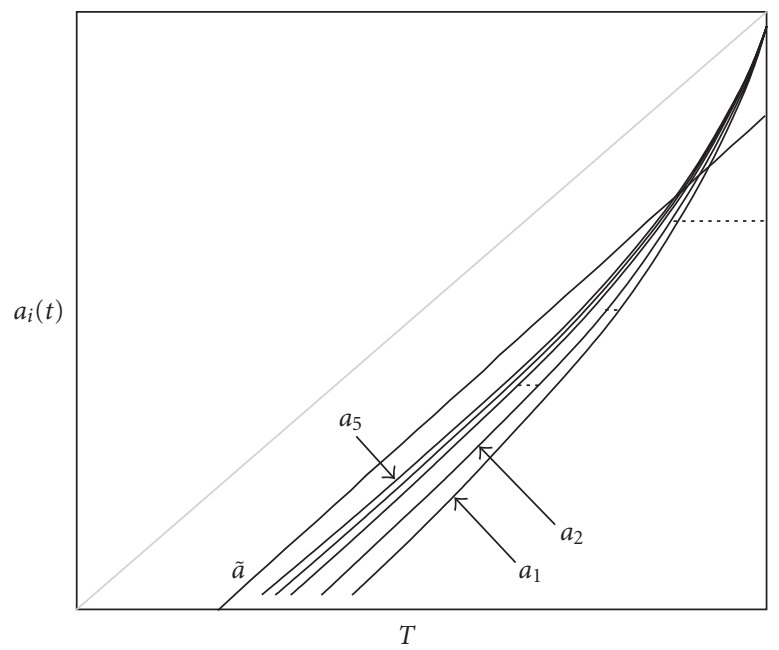

Figure 4.1. The trajectories $a_{i}, i=1,2,3, \ldots$, can keep the zero value of the OP gradient and are the only interior parts of the optimal trajectory $a$.

If $I^{\prime}(a ; t) \equiv 0, t \in \Delta \subset\left[t_{0}, T\right]$, then the functions $a(t)$ and $a^{-1}(t)$ satisfy (4.3) on $\Delta$. Since $a(t)<t$, then $a^{-1}(t)>t$ and expression (4.3) is a recurrent relation from the right to the left.

It appears that there is a discrete set of special trajectories $a_{i}, i=1,2, \ldots$, such that a function $a(t)$ should coincide with one of the trajectories $a_{i}(t)$ on $\Delta \subset\left[t_{0}, T\right]$ in order to produce $I^{\prime}(a ; t) \equiv 0$ at $t \in \Delta$. Namely, knowing $a(t) \equiv a(\Theta), t \in(\Theta, T]$, we can determine $a_{1}(t)$ from $(4.3)$ on the interval $[a(\Theta), \Theta]$, then determine $a_{2}(t)$ on the interval $\left[a_{1}(a(\Theta)), a(\Theta)\right]$, and so on. The trajectories $a_{i}(t)$ depend only on the constant $T$ and functions $\beta, \lambda, \rho$. Several first trajectories $a_{i}(t)$ calculated at $T=40, c_{1}=0.47, c_{2}=0.47$, $c_{3}=0.5, \beta_{0}=1$, and $\lambda_{0}=1.9$ are shown on Figure 4.1 with solid lines. At large $i$ and $T-t$, they are close to the solution $\tilde{a}$ of (3.7) on the infinite interval $\left[t_{0}, \infty\right)$, indicated by a gray line.

Lemma 4.2. The trajectories

$$
\begin{aligned}
& a_{1}(t)=t+\frac{1}{c_{1}} \ln \left\{\frac{1-\lambda_{0}}{\beta_{0}\left(c_{3}-c_{2}\right) e^{\left(c_{2}-c_{1}\right) t}+\left(c_{1} / c_{3}\right)\left[e^{-c_{3}(T-t)}-1\right]}\right\}, \quad t<T, \\
& a_{i+1}(t)=t+\frac{1}{c_{1}} \ln \left\{\frac{1-\lambda_{0}}{\beta_{0}\left(c_{3}-c_{2}\right) e^{\left(c_{2}-c_{1}\right) t}+\left(c_{1} / c_{3}\right)\left[e^{-c_{3}\left(a_{i}^{-1}(t)-t\right)}-1\right]}\right\}, \quad t<T, i=2,3, \ldots,
\end{aligned}
$$

have the following properties:

(1) if $I^{\prime}\left(a_{i} ; t\right)$ is constant at $t \in\left[t^{\prime}, t^{\prime \prime}\right], t^{\prime \prime} \leq T$, then $I^{\prime}\left(a_{i+1} ; t\right)$ is constant at $t \in\left[a_{i}\left(t^{\prime}\right)\right.$, $\left.a_{i}\left(t^{\prime \prime}\right)\right], t^{\prime \prime} \leq T, i \geq 1$

(2) $a_{i}(t)<a_{i+1}(t), t \in\left[t_{0}, T\right]$; 
(3) $d a_{i}(t) / d t>1, t \in\left[t_{0}, T\right]$, at $c_{1} \geq c_{2}$;

(4) $a_{1}(t) \rightarrow t+\ln \left[1-\lambda_{0} / \beta_{0}\left(c_{3}-c_{2}\right) \exp \left(c_{2}-c_{1}\right) t-c_{1} / c_{3}\right] / c_{1}$ as $T-t \rightarrow \infty$.

The proof follows from the analysis of the recurrent relation (4.3).

So, if a function $a$ satisfies equation $I^{\prime}(a ; t) \equiv 0$ on the interval $\left[t_{0}, T\right]$ with the initial condition (4.1) at the right end $(\Theta, T]$, it should coincide with the trajectories $a_{i}(t), i=$ $1,2,3, \ldots$, on the intervals $[a(\Theta), \Theta],[a(a(\Theta)), a(\Theta)],[a(a(a(\Theta))), a(a(\Theta))], \ldots$, and jump from $a_{i}(t)$ to $a_{i+1}(t)$. As follows from (4.5) and Figure 4.1, such a solution is discontinuous. Next, we use this solution to build a continuous quasisolution to the OP.

4.2. Structure of the OP solutions. We define the quasisolution to OP (2.1)-(2.4) as a continuous monotonic function $a_{q}(t), t \in\left[t_{0}, T\right]$, that satisfies the extremum condition (3.5) and does not necessarily satisfy the initial condition $a\left(t_{0}\right)=a_{0}$ in (2.4).

We assume here and thereafter that

$$
P^{\prime}(t) \geq 0, \quad t \in\left[t_{0}, T\right]
$$

This condition ensures that the quasisolution $a_{q}$ (if it exists) does not depend on $m$. Indeed, in view of (2.4),

$$
m(a(t)) a^{\prime}(t)=m(t)-P^{\prime}(t) \geq 0
$$

for any admissible $m$. So, the boundary-valued regime $m(t)=m_{\min }(t)$ at $\left[t_{1}, t_{2}\right] \subset\left[t_{0}, T\right]$ means $a^{\prime}(t) \equiv 0, a(t) \equiv a\left(t_{1}\right)$, and $m(t)=P^{\prime}(t) \geq 0$ for $t \in\left[t_{1}, t_{2}\right]$. Hence, the $a_{\min }(t)$, $t \in\left[t_{1}, t_{2}\right]$, depends on the value $a_{\min }\left(t_{1}\right)$ only and does not depend on $m_{\min }$.

Because of Lemma 4.2, we can separate the interval $\left[t_{0}, T\right]$ of any finite length into the parts $\left[a_{q}(\Theta), \Theta\right],\left[a_{q}\left(a_{q}(\Theta)\right), a_{q}(\Theta)\right],\left[a_{q}\left(a_{q}\left(a_{q}(\Theta)\right)\right), a_{q}\left(a_{q}(\Theta)\right)\right], \ldots$, and assign the intended quasisolution $a_{q}$ to the trajectories $a_{i}(t), i=1,2,3, \ldots$. To obtain a continuous quasisolution, we connect the separate pieces of $a_{i}$ with boundary-valued trajectories $a_{\min }$ as it is illustrated on Figure 4.1 with the dashed lines. The full implementation of the explained scheme is provided below.

LEMMA 4.3 (on OP quasisolution). Under conditions (3.8) and (4.6), there exists a quasisolution $a_{q}(t)$ to the finite-horizon $O P(2.1)-(2.4)$ :

$$
a_{q}(t)=\left\{\begin{array}{ll}
a_{i}\left(\alpha_{i}\right), & I^{\prime}(t)<0, t \in\left(\alpha_{i}, \beta_{i}\right) \\
a_{i}(t), & I^{\prime}(t)=0, t \in\left[\beta_{i+1}, \alpha_{i}\right),
\end{array} \quad i=1,2, \ldots, t \in\left[t_{0}, T\right),\right.
$$

where the parameters $\alpha_{i}, \beta_{i}, i=1,2,3, \ldots$, are uniquely determined, $\beta_{i+1}<\alpha_{i}, \alpha_{i}<\beta_{i}, \beta_{1}=T$, and the trajectories $a_{i}, i=1,2,3, \ldots$, are determined in Lemma 4.2 .

Proof. The construction of the quasisolution $a_{q}$ starts from the right end $T$ of the horizon $\left[t_{0}, T\right]$. In view of Lemma 4.1, the gradient $I^{\prime}\left(a_{q} ; t\right)<0$ on some "zero-investment" inter$\operatorname{val}(\Theta, T]$ to the left of $T$. Hence, $a_{q}(t)$ is minimum possible, $a_{q}(t)=a_{\min }(t) \equiv a_{q}(T)$, and $a_{q}^{-1}(t) \equiv T, t \in[\Theta, T]$. After increasing $I^{\prime}\left(a_{q} ; t\right)$ up to zero at $t=\Theta$, we keep $I^{\prime}\left(a_{q} ; t\right)=0$ to the left of $\Theta$. Lemma 4.2 shows that the only way to implement this is to keep $a_{q}(t)$ on the curve $a_{1}(t)$ at $t<\Theta$. So, we need to find the point $\left(\Theta, a_{1}(\Theta)\right)$ on the curve $a_{1}(t)$ 
that satisfies $I^{\prime}\left(a_{q} ; \Theta\right)=0$. To show that such $\Theta$ exists, we investigate the asymptotic of function $I^{\prime}\left(a_{q} ; \Theta\right)$. The substitution of $a_{q}(t)$ and $a_{q}^{-1}(t), t \in[\Theta, T]$, into (4.2) leads to

$$
\begin{aligned}
I^{\prime}\left(a_{q} ; \theta\right) & =\int_{\theta}^{T} e^{-c_{3} \tau}\left[e^{c_{1} \theta}-e^{c_{1} a(\theta)}\right] d \tau-\frac{\lambda_{0}}{\beta_{0} e^{\left(-c_{3}+c_{2}\right) \theta}} \\
& =e^{\left(-c_{3}+c_{1}\right) \theta}\left\{\frac{\left[1-e^{c_{1}[a(\theta)-\theta]}\right]\left[1-e^{-c_{3}(T-\theta)}\right]}{c_{3}}-\frac{\lambda_{0}}{\beta_{0} e^{\left(c_{2}-c_{1}\right) \theta}}\right\} .
\end{aligned}
$$

Substituting the asymptotic expression of $a_{1}(t)$ at $T-t \rightarrow \infty$ from Lemma 4.2 for $a(\Theta)$, we obtain that

$$
\begin{aligned}
I^{\prime}\left(a_{q} ; \theta\right)=e^{\left(-c_{3}+c_{1}\right) \theta}\{[ & \left.\left.\frac{\lambda_{0}}{\beta_{0}\left(c_{3}-c_{2}\right) e^{\left(c_{2}-c_{1}\right) \theta}}-\frac{\lambda_{0}}{\beta_{0} c_{1}}+\frac{c_{1}}{c_{3}}\right] \frac{\left[1-e^{-c_{3}(T-\theta)}\right]}{c_{3}}-\frac{\lambda_{0}}{\beta_{0} e^{\left(c_{2}-c_{1}\right) \theta}}\right\} \\
= & \frac{e^{\left(-c_{3}+c_{1}\right) \theta}}{c_{3}}\left\{\left[\frac{\lambda_{0}}{\beta_{0} c_{3} e^{\left(c_{2}-c_{1}\right) \theta}}+\frac{c_{1}}{c_{3}}\left(\frac{1-\lambda_{0}}{\beta_{0} c_{3}}\right)\right]\left[1-e^{-c_{3}(T-\theta)}\right]-\frac{\lambda_{0}}{\beta_{0} e^{\left(c_{2}-c_{1}\right) \theta} c_{3}}\right\} \\
=\frac{e^{\left(-c_{3}+c_{1}\right) \theta}}{c_{3}}\{[ & {\left[-\frac{c_{2}-c_{1}}{c_{3}}+\frac{c_{2}}{c_{3}}\left(1-\frac{\lambda_{0}}{\beta_{0}} c_{3} e^{\left(c_{2}-c_{1}\right) \theta}\right)\right]\left[1-e^{-c_{3}(T-\theta)}\right] } \\
& \left.-\frac{\lambda_{0}}{\beta_{0}} e^{\left(c_{2}-c_{1}\right) \theta} c_{3} e^{-c_{3}(T-\theta)}\right\} .
\end{aligned}
$$

Using the inequalities in (3.8), we obtain $I^{\prime}\left(a_{q} ; \Theta\right)>0$ for $T-\Theta \gg 1$. Since $I^{\prime}\left(a_{q} ; T\right)<0$ and $I^{\prime}\left(a_{q} ; \Theta\right)$ is continuous, at $T-t_{0} \gg 1$ a unique moment $\alpha_{1}=\Theta$ exists that satisfies the equality $I^{\prime}\left(a_{q} ; \Theta\right)=0$.

We construct the next piece of $a_{q}$ on the interval $\left[\alpha_{2}, \alpha_{1}\right]$. Because of the symmetry of inverse functions, the inverse $a_{q}^{-1}(t), t \in\left(a_{1}\left(a_{1}(\Theta)\right), T\right]$, is already defined by $a_{q}(t)$, $t \in\left(a_{1}(\Theta), T\right]$. We put $a_{q}(t)=a_{1}(t)$ to keep $I^{\prime}\left(a_{q} ; t\right) \equiv 0$ to the left of $\alpha_{1}$ on some interval $\left[\beta_{2}, \alpha_{1}\right]$. So, the trajectory $a_{q}(t)$ has to leave $a_{1}(t)$ at some point $\beta_{2}$, before $a_{q}^{-1}(t)$ jumps from $T$ to $a_{1}^{-1}(t)$ at $t=a_{1}(\Theta)$. To the left of $\beta_{2}, a_{q}(t)$ follows the boundary minimum trajectory $a_{q}(t)=a_{q}\left(\beta_{2}\right)=a_{1}\left(\beta_{2}\right)$ until it reaches the second line $a_{2}(t)$ at some point $\alpha_{2}<\beta_{2}$. The points $\alpha_{2}$ and $\beta_{2}$ are found from the condition $I^{\prime}\left(a_{q} ; \alpha_{2}\right)=0$ on the new curve $a_{2}(t)$. To show that the point $\alpha_{2}$ exists, we estimate that the gradient $I^{\prime}\left(a_{q} ; t\right)>0$ at $\alpha_{2}^{\prime \prime}=a_{1}(\Theta)$ and $I^{\prime}\left(a_{q} ; t\right)<0$ at the point $\alpha_{2}^{\prime}<\alpha_{2}^{\prime \prime}$ such that $\beta_{2}^{\prime}=a_{1}(\Theta)$ and $a_{2}\left(\alpha_{2}^{\prime}\right)=$ $a_{1}\left(\beta_{2}^{\prime}\right)$. Because of the continuity of $I^{\prime}\left(a_{q} ; t\right)$ in $t$, a unique moment $\alpha_{2}, \alpha_{2}^{\prime}<\alpha_{2}<\alpha_{2}^{\prime \prime}$, exists such that $I^{\prime}\left(a_{q} ; \alpha_{2}\right)=0$.

We show that $I^{\prime}\left(a_{q} ; t\right)$ is less than 0 when the quasisolution $a_{q}(t)$ leaves the curve $a_{1}(t)$ at $t=\beta_{2}$ and until it reaches $a_{2}(t)$ at $t=\alpha_{2}$. By construction, the gradient $I^{\prime}\left(a_{q} ; t\right) \equiv 0$ on interval $\left[\beta_{2}, \alpha_{1}\right]$. We investigate its derivative $d\left[I^{\prime}\left(a_{q} ; t\right)\right] / d t$ in $t$ :

$$
\begin{aligned}
\frac{d\left[I^{\prime}(a ; t)\right]}{d t}= & -\frac{c_{1}}{c_{3}}\left[e^{-c_{3} a^{-1}(t)}-e^{-c_{3} t}\right] e^{c_{1} t}+\left[e^{c_{1} a(t)}-e^{c_{1} t}\right] e^{-c_{3} t} \\
& +\lambda_{0}\left(c_{3}-c_{2}\right) e^{-\left(c_{3}-c_{2}\right) t}, \quad t \in\left[t_{0}, T\right)
\end{aligned}
$$


At the beginning, we consider a small neighborhood of the instant $t=\beta_{2}$. Here $a_{q}(t)>$ $a_{1}(t)$ at $t<\beta_{2}$, hence $d\left[I^{\prime}\left(a_{q} ; t\right)\right] / d t>d\left[I^{\prime}\left(a_{1} ; t\right)\right] / d t=0$ in view of the last formula. Similarly, $d\left[I^{\prime}\left(a_{q} ; t\right)\right] / d t<d\left[I^{\prime}\left(a_{2} ; t\right)\right] / d t=0$ at some neighborhood $t>\alpha_{2}$. Therefore, $I^{\prime}\left(a_{q}\right.$; $t)<0$ on interval $\left(\alpha_{2}, \beta_{2}\right)$.

The previous part contains a complete iteration in constructing the quasisolution $a_{q}$. At the beginning, $a_{q}(t)=a_{1}(t), t \in\left(\beta_{2}, \alpha_{1}\right)$, hence $a_{q}^{-1}(t)=a_{1}^{-1}(t)$ at $t<a_{1}\left(\beta_{2}\right)$. According to Lemma 4.2, the new curve is $a_{2}(t)$ on some interval to the left of $\beta_{2}$. The trajectory $a_{q}$ is minimum possible $a_{q}(t)=a_{q}\left(\beta_{2}\right)=a_{1}\left(\beta_{2}\right)$ until it intersects $a_{2}$ at some point $\alpha_{2}<\beta_{2}$. Then the corresponding $a_{q}^{-1}$ may be found, the iteration may be repeated, and so on. The "switch" points $\alpha_{i}, \beta_{i}, a_{i}\left(\alpha_{i}\right)=a_{i-1}\left(\beta_{i}\right), i=1,2, \ldots$, where the quasisolution $a_{q}(t)$ leaves the old curve $a_{i}(t)$ for the new one, are uniquely determined from the equation $I^{\prime}\left(a_{q} ; \alpha_{i}\right)=0$ on the new curve $a_{i+1}$.

Finally, we verify that the quasisolution $a_{q}$ satisfies the extremum conditions (3.5). Namely, $a_{q}(t), t \in\left[t_{0}, T\right]$, is constructed in such a way that $I^{\prime}\left(a_{q} ; t\right)<0$ on $\left(\alpha_{i}, \beta_{i}\right)$ or where $a_{q}=a_{\min } \equiv$ const, and $I^{\prime}\left(a_{q} ; t\right)=0$ on $\left(\beta_{i+1}, \alpha_{i}\right)$ or where $a_{q} \equiv a_{i}$.

If a quasisolution $a_{q}$ exists, then the optimal trajectory $a^{*}$ will coincide with it except for an initial finite interval $\left[t_{0}, \mu\right)$. At the initial interval $\left[t_{0}, \mu\right)$, the OP solution will be boundary-valued: $m^{*} \equiv m_{\min }$ or $m^{*} \equiv M$. The corresponding $m^{*}(t), t \in\left[t_{0}, T\right]$, is defined from (2.2) and always depends on the initial condition $m_{0}$.

The explicit formula (4.8) for the OP quasisolution $a_{q}$ allows us to prove the following result.

Theorem 4.4 (on the structure of the OP solution). Under conditions (3.8) and (4.6), OP (2.1)-(2.4) has the unique solution $\left(m^{*}, a^{*}\right)$ of the following form:

$$
\begin{gathered}
m^{*}(t)= \begin{cases}m_{\min }(t) \text { or } M(t), & t \in\left[t_{0}, \mu\right), \\
m_{q}(t), & t \in[\mu, T),\end{cases} \\
a^{*}(t)= \begin{cases}a_{\mu}(t), & t \in\left[t_{0}, \mu\right), \\
a_{q}(t), & t \in[\mu, T),\end{cases}
\end{gathered}
$$

where

$$
a_{\mu}(t)= \begin{cases}a_{\min }(t) & \text { if } a_{0}>\tilde{a}\left(t_{0}\right), \\ a_{\max }(t) & \text { if } a_{0}<\tilde{a}\left(t_{0}\right),\end{cases}
$$

and $a_{q}$ is the quasisolution determined by Lemma 4.3. The function $m_{q}$ is found from (2.2) at $a \equiv a_{q}$, the functions $a_{\min }$ and $a_{\max }$ are defined from (2.2) and correspond to the minimum $m \equiv m_{\min }$ and maximum $m \equiv M$, and the instant $\mu$ is determined from the condition $a_{\mu}(\mu)=a_{q}(\mu)$. 
Proof. The proof consists of two steps: (a) the construction of an admissible solution $\left(m^{*}, a^{*}\right)$ and (b) the verification of its optimality. During the first step, the optimal solution $a^{*}$ is obtained by the adjustment of the quasisolution $a_{q}$ to the initial conditions (2.4). Two cases are possible.

Case 1. $a_{0}>a_{q}\left(t_{0}\right)$. We choose $m^{*}(t)=m_{\min }(t)$ at $t>t_{0}$ and move on the corresponding $a_{\min }(t) \equiv a_{0}$ until it crosses the trajectory $a_{q}$. Then the point of interception is $\mu$ and the line of movement is $a_{\mu}(t), t \in\left[t_{0}, \mu\right]$. According to (4.7), $a_{\mu}(t)=a_{0}, t \in\left[t_{0}, \mu\right]$, and the corresponding gradient

$$
\begin{aligned}
I^{\prime}\left(a^{*} ; t\right)= & \int_{t}^{a^{*^{-1}}(t)} e^{-c_{3} \tau}\left[e^{c_{1} t}-e^{c_{1} a^{*}(\tau)}\right] d \tau-\lambda_{0} e^{\left(-c_{3}+c_{2}\right) t} \\
= & \int_{t}^{a_{q}^{-1}(t)} e^{-c_{3} \tau}\left[e^{c_{1} t}-e^{c_{1} a_{q}(\tau)}\right] d \tau-\lambda_{0} e^{\left(-c_{3}+c_{2}\right) t} \\
& +\int_{t}^{\mu} e^{-c_{3} \tau}\left[e^{c_{1} a_{q}(\tau)}-e^{c_{1} a_{0}}\right] d \tau \\
= & \int_{t}^{\mu} e^{-c_{3} \tau}\left[e^{c_{1} a_{q}(\tau)}-e^{c_{1} a_{0}}\right] d \tau<0, \quad t \in\left[t_{0}, \mu\right],
\end{aligned}
$$

that is, the pair $\left(a^{*}, m^{*}\right)$ satisfies the extremum conditions (3.5) at $t \in\left[t_{0}, \mu\right]$. Later on at $t>\mu$, the solution $a^{*}(t)$ coincides with the quasisolution $a_{q}(t)$. The corresponding $m^{*}(t), t \in[\mu, T]$, is determined from (4.7), hence, it will be 0 on $\left(\alpha_{i}, \beta_{i}\right)$ where $I^{\prime}\left(a^{*} ; t\right)<0$ and an internal value between $m_{\min }$ and $M$ from the domain (2.3) on $\left(\beta_{i+1}, \alpha_{i}\right)$ where $I^{\prime}\left(a^{*} ; t\right)=0, i=1,2,3, \ldots$ Therefore, according to Lemma 4.2 , the pair $(4.12)$ is a solution to OP $(2.1)-(2.4)$.

Case 2. $a_{0}<a_{q}\left(t_{0}\right)$ is investigated similarly. In this case $m(t)=M$ brings $a_{\mu}(t)$ up to the point of its interception with the trajectory $a_{q}$ and $I^{\prime}\left(a^{*} ; t\right)>0$ on $t \in\left[t_{0}, \mu\right)$.

The dynamics of OP solution $\left(a^{*}, m^{*}\right)$ and the corresponding gradient $I^{\prime}(t)$ are depicted in Figure 4.2 for the case $P^{\prime}(t)=0$. The restriction $P^{\prime}=0$ is selected for simplicity only (then two boundary-valued regimes $a_{\min }^{\prime} \equiv 0$ and $m_{\min } \equiv 0$ coincide).

Theorem 4.4 shows that the irregularities in the optimal controls $m^{*}$ and $a^{*}$ are caused by the initial and final conditions of the OP. First, the "imperfect" initial condition $a\left(t_{0}\right)=$ $a_{0} \neq \tilde{a}\left(t_{0}\right)$ on the left end $t=t_{0}$ of $\left[t_{0}, T\right]$ causes the appearance of an initial boundaryvalued section $m(t) \equiv 0$ or $M, t \in\left[t_{0}, \mu\right]$, in the optimal trajectory $m^{*}$. The control $m^{*}(t)$ is determined by (2.2) as $m^{*}(t)=P^{\prime}(t)+m\left(a^{*}(t)\right) d a^{*} / d t$ from the left to the right, starting with the initial condition (2.4). This formula reproduces the jump in $m^{*}$ throughout the whole horizon $\left[t_{0}, T\right]$ (when we reach the interval $\left[a^{-1}\left(t_{0}\right), a^{-1}(\mu)\right]$, later on the inter$\operatorname{val}\left[a^{-1}\left(a^{-1}\left(t_{0}\right)\right), a^{-1}\left(a^{-1}(\mu)\right)\right]$, and so on). This phenomenon was earlier analyzed in [2].

Secondly, the optimal trajectory $a^{*}$ also has irregular sections $\left[\alpha_{i}, \beta_{i}\right]$ where $a^{*^{\prime}}(t) \equiv 0$. They represent the impact of the zero-investment period $\left(\alpha_{1}, T\right]$ at the right end of $\left[t_{0}, T\right]$ on optimal trajectories. When we reach such a section, then $m^{*}=m_{\min }=P^{\prime}$. Thus, the optimal control $m^{*}$ has two groups of the replacement echoes on the planning horizon $\left[t_{0}, T\right]$ : (a) the echoes caused by the "imperfect" initial condition $a\left(t_{0}\right)=a_{0} \neq \tilde{a}\left(t_{0}\right)$ at $t=t_{0}$; (b) the "zero-investment" echoes caused by the "zero-investment period" $\left(\alpha_{1}, T\right]$ 


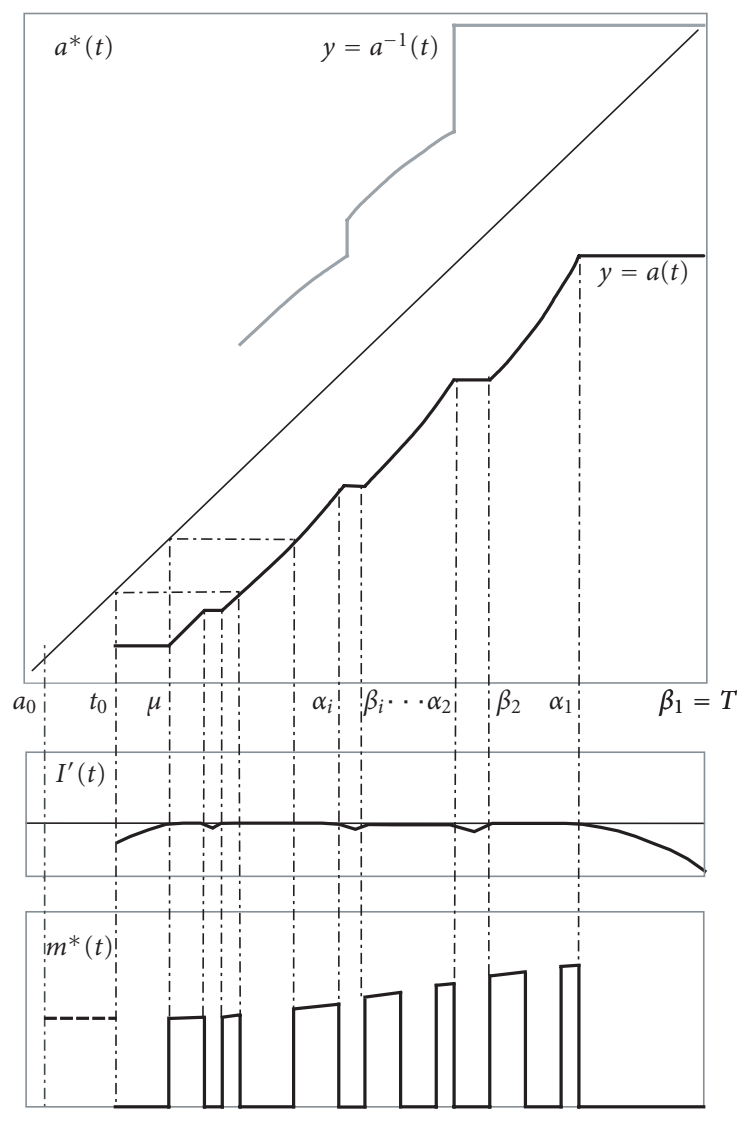

Figure 4.2. The solution $a$ and $m$ and the gradient of OP (2.1)-(2.4).

at the right end of $\left[t_{0}, T\right]$. The "zero-investment" echoes propagate backward throughout the whole horizon $\left[t_{0}, T\right]$ starting from the right end of $\left[t_{0}, T\right]$.

\section{Conclusion}

The constructed exact solution to Solow VCM considerably develops the mathematical theory of VCMs. Investigation of applied OPs usually involves a combination of analytic, approximate, and simulation methods. The construction of exact solutions is important to every applied mathematical problem, especially to nonlinear optimal control problems because of their high analytic and computational complexity. Even the existence of a solution is usually an open question and the exact solution automatically solves the existence problem.

The established structure of the exact OP solutions provides a new insight into the optimal dynamics of the capital renovation process. An important feature of this process is that the optimal trajectories do not possess irregularities of an arbitrary small length similar to "vibration controls" or generalized functions (see $[11,18]$ and others). While 
such behavior of optimal controls is natural for technical applications, it causes essential problems in economic interpretation.

\section{Acknowledgment}

The authors are extremely impressed by the high mathematical culture of an anonymous referee whose remarks helped to improve the presentation of certain issues.

\section{References}

[1] J. Benhabib and A. Rustichini, Vintage capital, investment, and growth, J. Econom. Theory 55 (1991), no. 2, 323-339.

[2] R. Boucekkine, M. Germain, and O. Licandro, Replacement echoes in the vintage capital growth model, J. Econom. Theory 74 (1997), no. 2, 333-348.

[3] J. Campbell, Entry, exit, embodied technology, and business cycles, Rev. Econ. Dynam. 1 (1998), no. 2, 371-408.

[4] T. Cooley, J. Greenwood, and M. Yorukoglu, The replacement problem, J. Monet. Econ. 40 (1997), no. 3, 457-499.

[5] C. Corduneanu, Integral Equations and Applications, Cambridge University Press, Cambridge, 1991.

[6] N. Hritonenko and Yu. Yatsenko, Integral-functional equations for optimal renovation problems, Optimization 36 (1996), no. 3, 249-261.

[7] Modeling and Optimization of the Lifetime of Technologies, Applied Optimization, vol. 4, Kluwer Academic Publishers, Dordrecht, 1996.

[8] - Turnpike theorems in an integral dynamic model of economic restoration, Cybernet. Systems Anal. 33 (1997), no. 2, 259-273.

[9] _ Mathematical Modeling in Economics, Ecology and the Environment, Applied Optimization, vol. 34, Kluwer Academic Publishers, Dordrecht, 1999.

[10] - Applied Mathematical Modelling of Engineering Problems, Applied Optimization, vol. 81, Kluwer Academic Publishers, Massachusetts, 2003.

[11] A. D. Ioffe and V. M. Tihomirov, Theory of Extremal Problems, Studies in Mathematics and Its Applications, vol. 6, North-Holland Publishing, Amsterdam, 1979.

[12] V. V. Ivanov and Ju. P. Jacenko, Problem of optimal control for integral dynamic models with controllable prehistory, System Modelling and Optimization (Budapest, 1985), Lecture Notes in Control and Inform. Sci., vol. 84, Springer, Berlin, 1986, pp. 350-357.

[13] B. Jovanovic, Vintage capital and inequality, Rev. Econ. Dynam. 1 (1998), no. 2, 497-530.

[14] J. M. Malcomson, Replacement and the rental value of capital equipment subject to obsolescence, J. Econom. Theory 10 (1975), no. 1, 24-41.

[15] R. T. Rockafellar, Convex Analysis, Princeton Mathematical Series, no. 28, Princeton University Press, New Jersey, 1970.

[16] R. M. Solow, J. Tobin, C. von Weizsacker, and M. Yaari, Neoclassical growth with fixed factor proportions, Rev. Econom. Stud. 33 (1966), 79-115.

[17] O. van Hilten, The optimal lifetime of capital equipment, J. Econom. Theory 55 (1991), no. 2, 449-454.

[18] J. Warga, Optimal Control of Differential and Functional Equations, Academic Press, New York, 1972.

[19] Yu. Yatsenko, Integral Models of Systems with Controllable Memory, Naukova Dumka, Kiev, 1991.

[20] , Volterra integral equations with unknown delay time, Methods Appl. Anal. 2 (1995), no. $4,408-419$. 
[21] Yu. Yatsenko and N. Hritonenko, Optimization in integral model of developing systems, Optimization 31 (1994), no. 2, 179-192.

Natali Hritonenko: Department of Mathematics, Prairie View A\&M University, Prairie View, TX 77446-4189, USA

E-mail address: natalia_hritonenko@pvamu.edu

Yuri Yatsenko: College of Business \& Economics, Houston Baptist University, 7502 Founder Road, Houston, TX 77074-3298, USA

E-mail address: yyatsenko@hbu.edu 


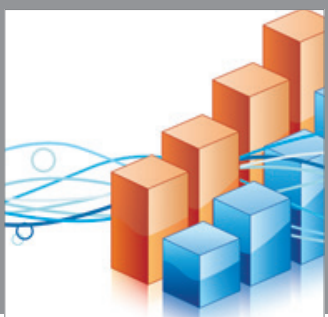

Advances in

Operations Research

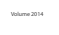

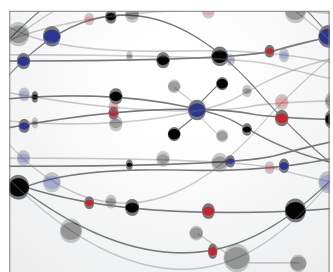

\section{The Scientific} World Journal
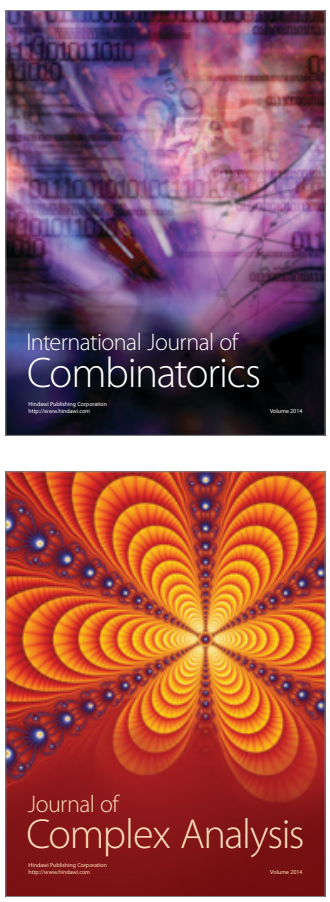

International Journal of

Mathematics and

Mathematical

Sciences
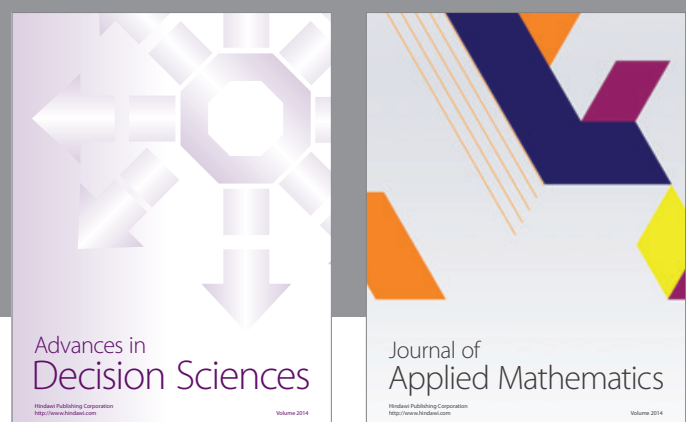

Journal of

Applied Mathematics
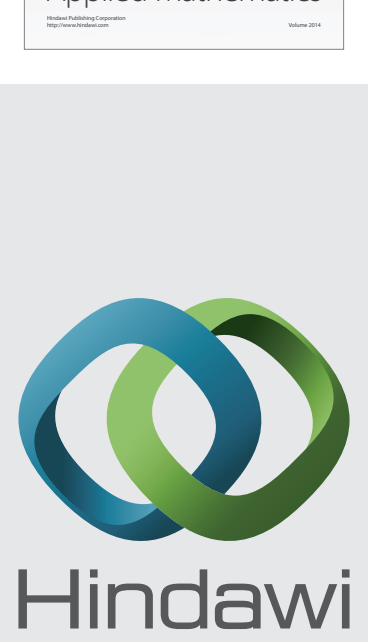

Submit your manuscripts at http://www.hindawi.com
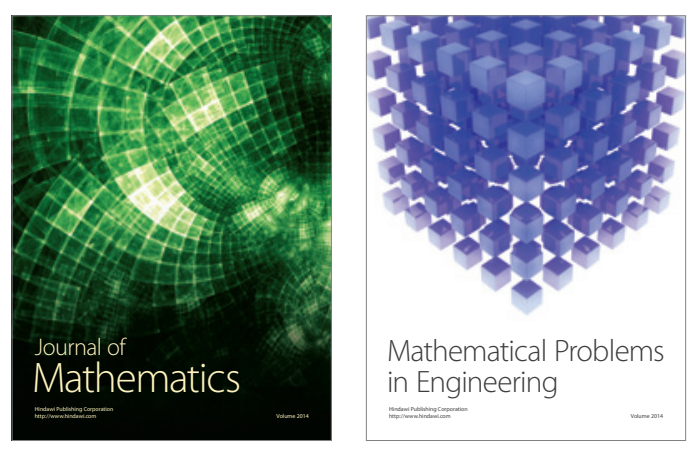

Mathematical Problems in Engineering
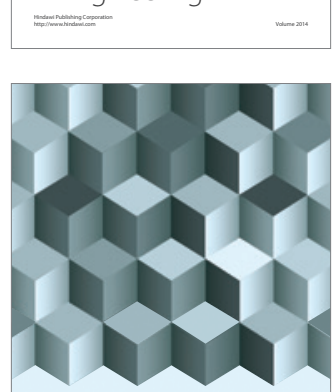

Journal of

Function Spaces
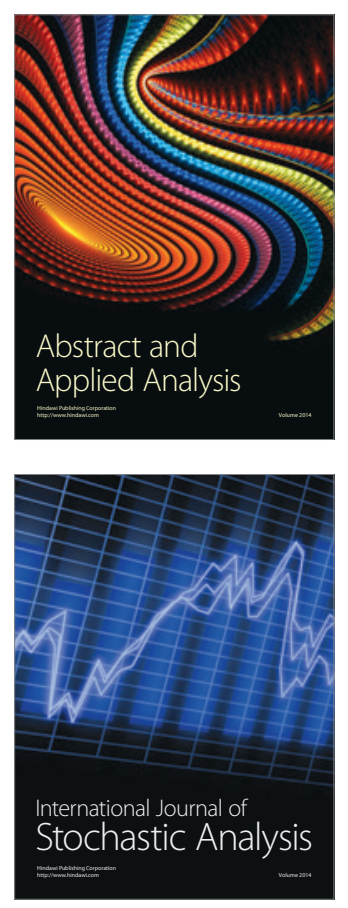

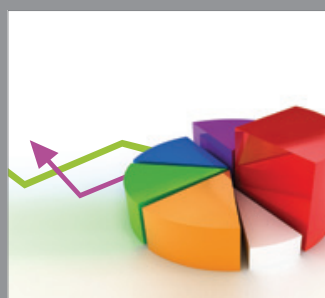

ournal of

Probability and Statistics

Promensencen
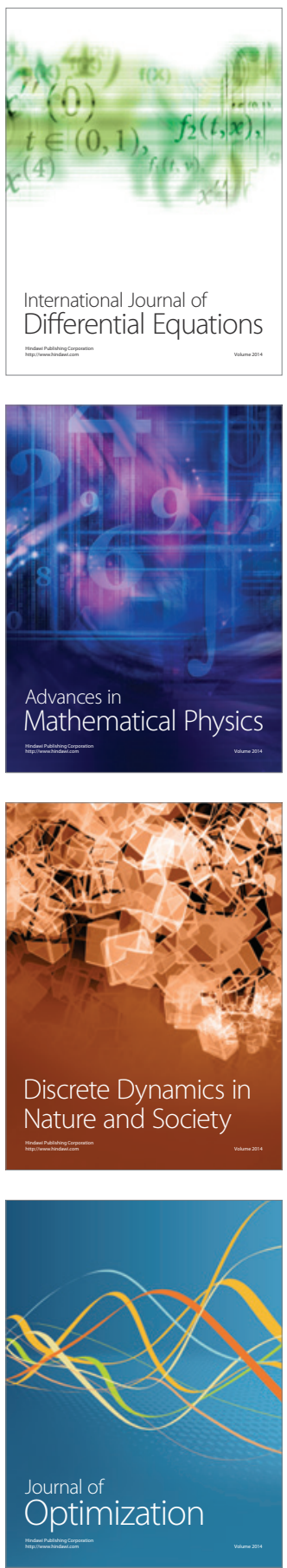